TRANSACTIONS OF THE

AMERICAN MATHEMATICAL SOCIETY

Volume 359, Number 6, June 2007, Pages 2483-2500

S 0002-9947(07)04063-9

Article electronically published on January 4, 2007

\title{
COARSE CLASSIFICATION OF CONSTANT MEAN CURVATURE CYLINDERS
}

\author{
J. DORFMEISTER AND S.-P. KOBAYASHI
}

\begin{abstract}
We give a coarse classification of constant mean curvature (CMC) immersions of cylinders into $\mathbb{R}^{3}$ via the loop group method. Particularly for this purpose, we consider double loop groups and a new type of "potentials" which are meromorphic 1-forms on Riemann surfaces.
\end{abstract}

\section{INTRODUCTION}

The goal of this paper is to give a coarse classification of immersions of cylinders into $\mathbb{R}^{3}$ of constant mean curvature (CMC) via the loop group method [13].

According to 13 one can construct every CMC-immersion of mean curvature $H \neq 0$ which does not only parametrize part of a sphere from a simply-connected domain $\mathfrak{D} \subset \mathbb{C}$ into $\mathbb{R}^{3}$ in four steps as follows:

Step 1: Choose any holomorphic $2 \times 2$-matrix differential form $\eta=A(z, \lambda) d z$, of which the diagonal elements are even functions of $\lambda \in \mathbb{C}^{*}$, the off-diagonal elements are odd functions of $\lambda \in \mathbb{C}^{*}$, and the powers of $\lambda$ are $\geq-1$. Assume $\operatorname{det} A_{-1} \neq 0$, where $A_{-1}$ is the coefficient matrix of the $\lambda^{-1}$ term of $\eta$.

Step 2: Solve the ODE $d C=C \eta$.

Step 3: Perform an Iwasawa splitting: $C=F W_{+}$, where $F=F(z, \bar{z}, \lambda)$ is unitary for all $z \in \mathfrak{D}, \lambda \in \mathbb{S}^{1}$, and $W_{+}$has a Fourier expansion relative to $\lambda$ without negative exponents.

Theorem 1.1 ([13]). $F$ is, for every fixed $\lambda \in S^{1}$, a frame of some immersion of constant mean curvature $H \neq 0$.

Step 4: Form $\Psi_{\lambda}(z)=-\frac{1}{2 H}\left\{\left(\frac{d}{d t} F\right) F^{-1}+\frac{i}{2} F\left(\begin{array}{cc}1 & 0 \\ 0 & -1\end{array}\right) F^{-1}\right\}$ (Sym-Bobenko Formula). Then $\Psi_{\lambda}$ is a CMC-immersion of mean curvature $H \neq 0$ from $\mathfrak{D}$ to $\mathbb{R}^{3} \cong s u(2)$. Moreover, every CMC-immersion of mean curvature $H \neq 0$ different from a Riemann sphere $\mathbb{S}^{2}$ can be obtained this way.

This is called the "generalized Weierstrass representation" of CMC-immersions. Obviously, the procedure outlined above invariably leads to a CMC-immersion. The only variable input parameters are the choice of $\eta$ and the initial condition for $C$. If one has a certain CMC-immersion in mind, then if $\eta$ is chosen "right", one can

Received by the editors December 7, 2004.

2000 Mathematics Subject Classification. Primary 53A10.

Key words and phrases. Constant mean curvature surfaces, loop groups.

The first author acknowledges support by DFG.

The second author was fully supported by DFG grant DO776/1.

(C)2007 American Mathematical Society Reverts to public domain 28 years from publication 
choose the initial condition $C\left(z_{0}, \lambda\right)=\mathrm{Id}$, where $z_{0} \in \mathfrak{D}$ is some fixed "base point". In general, however, one does not know precisely what $\eta$ to choose. Therefore, one chooses $\eta$ "of reasonable shape" and makes additional "adjustments" via the initial condition of $C$, to finally construct an immersion with specifically desired properties [9, 8.

At any rate, the choice of $\eta$ is important. It has been known since 13] that one can replace the holomorphic differential form $\eta$ by some meromorphic differential form $\xi$ of the form $\xi=\lambda^{-1} \hat{\xi}$, where $\hat{\xi}$ is meromorphic on $\mathfrak{D}$. Moreover if the initial condition at some base point $z_{0} \in \mathfrak{D}$ is $C\left(z_{0}, \lambda\right)=\mathrm{Id}$, then there is a bijective relation between the "normalized potentials" $\xi=\lambda^{-1} \hat{\xi}$ and the CMC-immersions $\left(\neq \mathbb{S}^{2}\right.$ and $\left.H \neq 0\right)$ from $\mathfrak{D}$ into $\mathbb{R}^{3}$.

Clearly, the ultimate goal is to understand in detail what data $\eta$ to choose for the construction of all CMC-immersions from some Riemann surface $\mathcal{M}$ to $\mathbb{R}^{3}$. (Note that a real 2-dimensional manifold $S$ which does admit an immersion into $\mathbb{R}^{3}$ of constant mean curvature carries a complex structure, i.e., $S$ is a Riemann surface.) It is thus natural to look for potentials with additional properties, which are at least necessary for immersions descending from the universal cover $\mathfrak{D}$ of $\mathcal{M}$ to $\mathcal{M}$.

For non-compact $\mathcal{M}$ it has been shown in $[9]$, 8 that the CMC-immersions from $\mathcal{M}$ to $\mathbb{R}^{3}$ can be obtained by the generalized Weierstrass representation outlined above from a holomorphic potential $\eta$ on $\mathcal{M}$, which is invariant under the fundamental group $\pi_{1}(\mathcal{M}) \hookrightarrow \operatorname{Aut}(\mathfrak{D})$, i.e., from a holomorphic differential $(1,0)$-form on $\mathcal{M}$.

In this paper we present a new type of potential, which does exist for every CMCimmersion, from $\mathfrak{D}$ to $\mathbb{R}^{3}$, where $\mathfrak{D}$ is the universal cover of some Riemann surface $\mathcal{M}$, and which is always invariant under at least one generator of the fundamental group $\pi_{1}(\mathcal{M})$ of $\mathcal{M}$. As an application we present a "coarse" classification of all CMC-cylinders. (We would like to note that in some special cases the above mentioned new type of potentials has already been used [19, 22.

More precisely, in Section 2, we will give the basic notation and results for later use. We will always use (matrix) loop groups and loop Lie algebra with coefficient functions in the Wiener algebra. In particular, we will use the two main splitting theorems for loop groups occurring in this paper: the Birkhoff decomposition and the Iwasawa decomposition. In Theorem 2.9 we will introduce a new type of meromorphic 1-form on a Riemann surface $\mathcal{M}$, called "meromorphic potentials". Such potentials are locally, away from poles, the same as "holomorphic potentials" defined in [13. Such potentials are useful when one wants to show that certain monodromy matrices are unitary. In Section 3, we introduce double loop groups, and embed the framing $F$ of a CMC-immersion into this double loop group. In Section 3.2 we associate with this a pair of potentials, where the first factor is the usual CMC-potential $\eta(z)$ and the second factor is $-\overline{\eta(z)}^{t}$. In Section 3.3 we generalize the setting of Section 3.2 considerably: we consider pairs $(\eta(z), \tau(w))$ where $(z, w) \in \mathfrak{D} \times \overline{\mathfrak{D}}$, and $\overline{\mathfrak{D}}$ denotes the complex conjugate of $\mathfrak{D}$. Also, here $\tau$ has in general no relation with $\eta$. We show that one can derive a generalized "frame" $U=U(z, w)$ in two independent variables. With $U$ one can associate complex surfaces by Sym type formulas. We plan to pursue this in more detail in a separate publication. Combining the considerations of Sections 3.2 and 3.3 we show in Section 3.4 that every frame $F(z, \bar{z}, \lambda)$ of some CMC-immersion is the 
restriction of some meromorphic "frame" $U(z, w, \lambda)$; see Theorem 3.2 . In Section 4. we will give the new type of meromorphic 1-form which is the restriction of a generalized potential defined in Section 3. called "skew-hermitian potential". Also, we will show how to uniquely determine this "skew-hermitian potential" from the CMC-immersion, and for the CMC-cylinder case, we will show the skew-hermitian potential is well defined on the surface. In the last section, we give a complete classification of all frame periodic CMC-immersions (which is defined in Section 5) using skew-hermitian potentials. Then we show how all CMC-cylinders are characterized among all frame periodic CMC-immersions. Finally, we give a new example of a CMC-cylinder by applying the theory presented in this paper.

\section{BASIC DEFINITIONS AND RESULTS}

2.1. Loop groups. In this chapter we introduce a loop group, a loop algebra and two splitting theorems. Let $C_{r}:=\{\lambda \in \mathbb{C}|| \lambda \mid=r\}$ be the circle of radius $r$ with $r \in(0,1]$, and let $D_{r}:=\{\lambda \in \mathbb{C}|| \lambda \mid<r\}$ be the open disk of radius $r$. We denote the closure of $D_{r}$ by $\overline{D_{r}}:=\{\lambda \in \mathbb{C}|| \lambda \mid \leq r\}$. Also, let $A_{r}=\{\lambda \in \mathbb{C}|r<| \lambda \mid<$ $1 / r\}$. This is an open annulus containing $S^{1}$. Let $\overline{A_{r}}$ denote the closure of $A_{r}$.

Furthermore, let $E_{r}=\{\lambda \in \mathbb{C}|r<| \lambda \mid\}$ be the exterior of the circle $C_{r}$. For any $r \in(0,1] \subset \mathbb{R}$, we consider the twisted loop algebra and loop group:

$$
\begin{aligned}
\Lambda_{r} s l(2, \mathbb{C})_{\sigma} & =\left\{\alpha: C_{r} \rightarrow \operatorname{sl}(2, \mathbb{C}) \mid \alpha \text { is continous and } \alpha(-\lambda)=\sigma_{3} \alpha(\lambda) \sigma_{3}\right\}, \\
\Lambda_{r} S L(2, \mathbb{C})_{\sigma} & =\left\{g: C_{r} \rightarrow S L(2, \mathbb{C}) \mid g \text { is continous and } g(-\lambda)=\sigma_{3} g(\lambda) \sigma_{3}\right\}
\end{aligned}
$$

where $\sigma_{3}=\left(\begin{array}{cc}1 & 0 \\ 0 & -1\end{array}\right)$.

We need to define special subgroups of $\Lambda S L(2, \mathbb{C})_{\sigma}$. First we consider the twisted $S U(2) r$-loop group:

$$
\begin{array}{r}
\Lambda_{r} S U(2)_{\sigma}=\left\{F(\lambda) \in \Lambda_{r} S L(2, \mathbb{C})_{\sigma} \mid F(\lambda) \in S U(2), \text { for all } \lambda \in S^{1},\right. \\
\left.F(\lambda) \text { extends holomorphically to } A_{r}\right\} .
\end{array}
$$

Note that the definition of $\Lambda_{r} S U(2)_{\sigma}$ implies that $F$ is continuous on $\overline{A_{r}}$ and holomorphic on $A_{r}$. Next, we define the twisted "plus $r$-loop group" and "minus $r$-loop group":

$$
\begin{array}{r}
\Lambda_{r, B}^{+} S L(2, \mathbb{C})_{\sigma}=\left\{W_{+} \in \Lambda_{r} S L(2, \mathbb{C})_{\sigma} \mid W_{+}(\lambda)\right. \text { extends holomorphically to } \\
\left.D_{r} \text { and } B(0) \in \boldsymbol{B}\right\} \\
\Lambda_{r, B}^{-} S L(2, \mathbb{C})_{\sigma}=\left\{W_{+} \in \Lambda_{r} S L(2, \mathbb{C})_{\sigma} \mid W_{+}(\lambda)\right. \text { extends holomorphically to } \\
\left.E_{r} \text { and } B(\infty) \in \boldsymbol{B}\right\}
\end{array}
$$

where $\boldsymbol{B}$ is a group of diagonal matrices in $S L(2, \mathbb{C})$. If $\boldsymbol{B}=\{\operatorname{Id}\}$ we write the subscript $*$ instead of $\boldsymbol{B}$, if $\boldsymbol{B}=\{$ all diagonal matrices $\}$ we abbreviate $\Lambda_{r, B}^{+} S L(2, \mathbb{C})_{\sigma}$ and $\Lambda_{r, B}^{-} S L(2, \mathbb{C})_{\sigma}$ by $\Lambda_{r}^{+} S L(2, \mathbb{C})_{\sigma}$ and $\Lambda_{r}^{-} S L(2, \mathbb{C})_{\sigma}$, respectively. From now on we will use the subscript $\boldsymbol{B}$ as above only if $\boldsymbol{B} \cap S U(2)=\{\mathrm{Id}\}$ holds, in particular if $\boldsymbol{B}$ is the group of all diagonal matrices with positive real entries. When $r=1$, we always omit the 1 . In order to make the above groups and algebras complex Banach Lie groups and Lie algebras, we restrict the occurring matrix coefficients 
to the "Wiener algebra" (see [17], page 5)

$$
\mathcal{A}=\left\{f(\lambda)=\sum_{n \in \mathbb{Z}} f_{n} \lambda^{n}: C_{r} \rightarrow \mathbb{C} ; \sum_{n \in \mathbb{Z}}\left|f_{n}\right|<\infty\right\} .
$$

We will assume from here on that all matrix coefficients are contained in the Wiener algebra $\mathcal{A}$. It is well known that the Wiener algebra is a Banach algebra relative to the norm $\|f\|=\sum\left|f_{n}\right|$, and that $\mathcal{A}$ consists of continous functions. Moreover, with coefficients in $\mathcal{A}$, the loop groups and loop algebras defined above are Banach Lie groups and Banach Lie algebras.

From [13], we quote the following two splitting theorems.

Theorem 2.1 (Birkhoff decomposition). For any $r \in(0,1]$, we have the disjoint union

$$
\Lambda_{r} S L(2, \mathbb{C})_{\sigma}=\bigcup \Lambda_{r}^{-} S L(2, \mathbb{C})_{\sigma} \cdot w_{n} \cdot \Lambda_{r}^{+} S L(2, \mathbb{C})_{\sigma},
$$

where $w_{n}=\left(\begin{array}{cc}\lambda^{n} & 0 \\ 0 & \lambda^{-n}\end{array}\right)$ if $n=2 k$ and $\left(\begin{array}{cc}0 & \lambda^{n} \\ -\lambda^{-n} & 0\end{array}\right)$ if $n=2 k+1$. The loops, for which $n=0$, form an open dense subset of $\Lambda_{r} S L(2, \mathbb{C})_{\sigma}$, and the multiplication map

$$
\Lambda_{r, *}^{-} S L(2, \mathbb{C})_{\sigma} \times \Lambda_{r}^{+} S L(2, \mathbb{C})_{\sigma} \longrightarrow \Lambda_{r} S L(2, \mathbb{C})_{\sigma}
$$

is an analytic diffeomorphism onto its image.

Theorem 2.2 (Iwasawa decomposition). For any $r \in(0,1]$ and each $\boldsymbol{B}$ of diagonal matrices of $S L(2, \mathbb{C})$, which satisfies $U(1) \cdot \boldsymbol{B}=\{$ all diagonal matrices $\}$ and $S U(2) \cap$ $\boldsymbol{B}=\{\mathrm{Id}\}$, the multiplication map

$$
\Lambda_{r} S U(2)_{\sigma} \times \Lambda_{r, B}^{+} S L(2, \mathbb{C})_{\sigma} \rightarrow \Lambda_{r} S L(2, \mathbb{C})_{\sigma}
$$

is a real analytic diffeomorphism onto.

2.2. Holomorphic and normalized potentials. Let $\Psi_{\lambda}: \mathfrak{D} \rightarrow \mathbb{R}^{3}, \lambda \in S^{1}$, be the associated family of (conformal) constant mean curvature $H=1 / 2$ immersions, where $\mathfrak{D}=$ disk in $\mathbb{C}$ or $\mathfrak{D}=\mathbb{C}$. Moreover, let $F(z, \lambda): \mathfrak{D} \rightarrow \Lambda S U(2)_{\sigma}, \lambda \in S^{1}$, be the extended framing of $\Psi_{\lambda}$ (see [6]). It is well known (see also the Introduction) that $\Psi_{\lambda}$ can be obtained from $F$ by the Sym-Bobenko Formula

$$
\Psi_{\lambda}=-\left(\frac{\mathrm{d}}{\mathrm{dt}} F \cdot F^{-1}+\frac{i}{2} F \sigma_{3} F^{-1}\right),
$$

where we have set $\lambda=e^{i \mathrm{t}}$.

Next we quote Lemma 4.5 in [13.

Theorem 2.3 (Existence of holomorphic potentials). Let $\Psi_{\lambda}: \mathfrak{D} \rightarrow \mathbb{R}^{3}$ be a CMCimmersion with $H=1 / 2$, and let $F \in \Lambda_{r} S U(2)_{\sigma}$ be the extended framing of $\Psi_{\lambda}$. Then there exists a holomorphic 1-form $\eta$ on $\mathfrak{D}$ of the form:

$$
\eta(z, \lambda)=\sum_{j \geq-1} \eta_{j}(z) \lambda^{j} d z
$$

where $\sum \eta_{j}(z) \lambda^{j} \in \Lambda$ sl $(2, \mathbb{C})_{\sigma}$, such that a holomorphic solution $C \in \Lambda_{r} S L(2, \mathbb{C})_{\sigma}$ of $d C=C \eta$ has an Iwasawa splitting $C=F \cdot W_{+}$, that is, with $F$ as given above and with $W_{+} \in \Lambda_{r, B}^{+} S L(2, \mathbb{C})_{\sigma}$.

We call any holomorphic solution $C \in \Lambda_{r} S L(2, \mathbb{C})_{\sigma}$ to $d C=C \eta, \eta$ as above, a holomorphic extended framing. Also from Theorem 4.10 in [13, we have the following. 
Theorem 2.4 (Existence of normalized potentials). We retain the assumptions of Theorem 2.3. Then there exists a meromorphic 1-form $\xi$ on $\mathfrak{D}$ of the form:

$$
\xi(z, \lambda)=\lambda^{-1}\left(\begin{array}{cc}
0 & f \\
Q / f & 0
\end{array}\right) d z,
$$

where $f$ is a non-vanishing meromorphic function and $Q$ is a holomorphic function such that there exists a meromorphic solution $g_{-} \in \Lambda_{r} S L(2, \mathbb{C})_{\sigma}$ to $d g_{-}=g_{-} \xi$ with Iwasawa splitting $g_{-}=F g_{+}$, that is, with $F$ as given above and with $g_{+} \in$ $\Lambda_{r, B}^{+} S L(2, \mathbb{C})_{\sigma}$.

2.3. Dressing and symmetries. Let $\mathcal{F}$ be the set of extended framings of CMCimmersions. For $F(z, \bar{z}, \lambda) \in \mathcal{F}$ and $h_{+} \in \Lambda_{r}^{+} S L(2, \mathbb{C})_{\sigma}$ we define

$$
h_{+}(\lambda) F(z, \bar{z}, \lambda)=\left(h_{+} \# F\right)(z, \bar{z}, \lambda) g_{+}(z, \bar{z}, \lambda),
$$

where $\left(h_{+} \# F\right)(z, \bar{z}, \lambda)$ is the unitary part of the unique Iwasawa decomposition in $\Lambda_{r} S L(2, \mathbb{C})_{\sigma}$ and $g_{+}$its positive part. Let $\mathcal{F}_{\text {Id }}$ be the set of normalized extended framings with base point $z_{0} \in \mathfrak{D}$ i.e., $F \in \mathcal{F}_{\text {Id }}$ if and only if $F\left(z_{0}, \bar{z}_{0}, \lambda\right)=$ Id. Then $h_{+}(\lambda)=h_{+}(\lambda) F\left(z_{0}, \bar{z}_{0}, \lambda\right)=\left(h_{+} \# F\right)\left(z_{0}, \bar{z}_{0}, \lambda\right) g_{+}\left(z_{0}, \bar{z}_{0}, \lambda\right)$ implies $h_{+} \# F\left(z_{0}, \bar{z}_{0}, \lambda\right)$ $=$ Id. Thus $h_{+} \# F$ is again in $\mathcal{F}_{\text {Id }}$. We will say that $h_{+} \# F$ is obtained from $F$ by dressing with $h_{+}$.

We will also define the dressing on the level of holomorphic extended framings. Let $C$ be the solution of $d C=C \eta$ in Theorem 2.3 with some initial condition $C\left(z_{0}, \lambda\right)=$ Id. Then we define

$$
\hat{C}=h_{+}(\lambda) \cdot C \cdot h_{+}^{-1}(\lambda),
$$

where $h_{+}(\lambda) \in \Lambda_{r}^{+} S L(2, \mathbb{C})_{\sigma}$. We will say that $\hat{C}$ is obtained from $C$ by dressing with $h_{+}$. To see how the surface is changed by dressing with $h_{+}$, one needs to perform an Iwasawa decomposition of $h_{+} F=\tilde{F} \tilde{W}_{+}$, where $C=F \cdot W_{+}$is the Iwasawa splitting of $C$. Then $\tilde{F} \in \Lambda_{r} S U(2)_{\sigma}$ is the frame for a new CMC-immersion. This change in the frame from $F$ to $\tilde{F}$ is non-trivial. Moreover, the associated change on the surface level is also non-trivial.

2.4. Invariant potentials and the monodromy problem. In this subsection, we will consider the construction of CMC-immersions with $H=1 / 2$ from surfaces with a non-trivial fundamental group. The essential point in the construction of CMC-immersion here is the "Monodromy problem". Before we state the definition of a monodromy matrix, we recall [10].

Proposition 2.5. Let $\mathcal{M}=\Gamma \backslash \mathfrak{D}$ be a connected Riemann surface with universal cover $\mathfrak{D}$ and Fuchsian group $\Gamma$ and let $\Psi: \mathcal{M} \rightarrow \mathbb{R}^{3}$ be a CMC-immersion. Let $\Psi_{\lambda}$ denote the associated family of $\Psi$ and let $F$ and $C$ denote an extended framing and a holomorphic extended framing, respectively. Then for every $\gamma \in \Gamma$ there exists some $\chi_{\gamma} \in \Lambda S U(2)_{\sigma}$ such that $\gamma^{*} C=\chi_{\gamma}(\lambda) C G_{+}$and $\gamma^{*} F=\chi_{\gamma}(\lambda) F k(z, \bar{z})$ for some $G_{+} \in \Lambda^{+} S L(2, \mathbb{C})$ and $k \in U(1)$.

More generally we have the following definition.

Definition 2.6. Let $\mathcal{M}=\Gamma \backslash \mathfrak{D}$ be a connected Riemann surface with universal cover $\mathfrak{D}$ and Fuchsian group $\Gamma$. Let $\eta$ or $\xi$ be a holomorphic potential or a normalized potential on $\mathfrak{D}$ as in Theorem 2.3 or Theorem 2.4 and let $C$ be a solution to $d C=C \eta$ or $d C=C \xi$. Also, let $F$ be the unitary part of the Iwasawa splitting of $C=F W_{+}$, and let $\gamma \in \Gamma$ be some deck transformation. A matrix 
$M_{\gamma} \in \Lambda_{r} S L(2, \mathbb{C})_{\sigma}\left(\right.$ resp. $\left.\Lambda_{r} S U(2)_{\sigma}\right)$ is called a monodromy matrix for $\gamma$ and $C$ (resp. $F)$ if $\gamma^{*} C=M_{\gamma} C G_{+}\left(\right.$resp. $\left.\gamma^{*} F=M_{\gamma} F k\right)$, for some $G_{+} \in \Lambda S L_{r}^{+}(2, \mathbb{C})_{\sigma}$ (resp. $k \in U(1)$ ).

Remark 2.7. Here the $k$ defined above is the change of coordinate of a CMC surface. Note that:

(1) If $\mathcal{M}$ is $\mathbb{C}$, then from [7, without loss of generality we can assume $k=\mathrm{Id}$.

(2) Moreover, if $\mathcal{M}$ is non-compact, then for the same argument above, we also can assume $k=\mathrm{Id}$.

(3) Actually $k$ is irrelevant for the resulting CMC surface, since $k$ goes away in the Sym- Bobenko Formula.

It will be particularly convenient to have $G_{+}=$Id. We have the following necessary and sufficient condition for such a monodromy.

Proposition 2.8. Let $\mathcal{M}=\Gamma \backslash \mathfrak{D}$ be a connected Riemann surface with universal cover $\mathfrak{D}$ and Fuchsian group $\Gamma$. Let $\eta$ be a holomorphic or normalized potential on $\mathfrak{D}$ and let $C$ be a solution of $d C=C \eta$. Then $\eta$ satisfies $\gamma^{*} \eta=\eta$ for some $\gamma \in \Gamma$ if and only if there exists a monodromy $M_{\gamma}$ of $C$ such that $\gamma^{*} C=M_{\gamma} C$.

Proof. " $\Rightarrow$ " Let $C$ be the solution to $d C=C \eta$, and let $\gamma^{*} C$ be the solution to $d\left(\gamma^{*} C\right)=\left(\gamma^{*} C\right)\left(\gamma^{*} \eta\right)=\left(\gamma^{*} C\right) \eta$ with $\left(\gamma^{*} C\right)\left(z_{0}, \lambda\right)=M_{\gamma} \in \Lambda_{r} S L(2, \mathbb{C})_{\sigma}$. Then the uniqueness of the solutions to ODE's implies $\gamma^{*} C=M_{\gamma} C$ (see [4]). The converse statement is clear.

We now introduce the notion of a "meromorphic potential" on a Riemann surface $\mathcal{M}$, which is locally equivalent to the notion of a "holomorphic potential" as described in Theorem 2.3. These two notions are, however, globally different, i.e., in general holomorphic potentials are only well defined on a universal cover of $\mathcal{M}$, while meromorphic potentials as defined below are always well defined on $\mathcal{M}$.

Theorem 2.9 (Existence of meromorphic potentials). Let $\mathcal{M}=\Gamma \backslash \mathfrak{D}$ be a connected Riemann surface with Fuchsian group $\Gamma$. Also, let $\Psi_{\lambda}: \mathcal{M} \rightarrow \mathbb{R}^{3}$ be a $\mathrm{CMC}$ immersion with $H=1 / 2$, and let $F \in \Lambda_{r} S U(2)_{\sigma}$ be the extended frame of $\Psi_{\lambda}$. Then there exists a meromorphic 1-form $\eta$ on $\mathcal{M}$ of the form:

$$
\eta(z, \lambda)=\sum_{j \geq-1} \eta_{j}(z) \lambda^{j} d z
$$

satisfying

$$
\gamma^{*} \eta=\eta \text { for all } \gamma \in \Gamma,
$$

such that there exists a meromorphic solution $C \in \Lambda_{r} S L(2, \mathbb{C})_{\sigma}$ to $d C=C \eta$ with Iwasawa splitting $C=F \cdot W_{+}$, that is, with $F$ as given above and with $W_{+} \in$ $\Lambda_{r, B}^{+} S L(2, \mathbb{C})_{\sigma}$.

Proof. We follow the proof of Theorem 3.2 in 9 . We would like to find a $W_{+} \in$ $\Lambda_{r}^{+} S L(2, \mathbb{C})_{\sigma}$ such that $C=F W_{+}$is meromorphic and satisfies $\gamma^{*} C=\chi_{\gamma}(\lambda) C$ for $\gamma \in \Gamma$. By Lemma 4.5 in [13], there exists $\tilde{W}_{+}: \mathfrak{D} \rightarrow \Lambda^{+} S L(2, \mathbb{C})_{\sigma}$ such that

$$
\tilde{C}=F \tilde{W}_{+}: \mathfrak{D} \rightarrow \Lambda S L(2, \mathbb{C})_{\sigma}
$$

is holomorphic. Hence

$$
\gamma^{*} \tilde{C}=\left(\gamma^{*} F\right)\left(\gamma^{*} \tilde{W}_{+}\right)=\chi_{\gamma}(\lambda) F k\left(\gamma^{*} \tilde{W}_{+}\right)=\chi_{\gamma}(\lambda) \tilde{C} \tilde{W}_{+}^{-1} k\left(\gamma^{*} \tilde{W}_{+}\right),
$$


where $P_{+}=\tilde{W}_{+}^{-1} k\left(\gamma^{*} \tilde{W}_{+}\right)$is holomorphic. Since $k$ satisfies the cocycle condition (see Theorem 2.3 in [7]), $P_{+}=P_{+}(\gamma, z)$ satisfies the cocycle condition

$$
P_{+}\left(\gamma_{2} \gamma_{1}, z\right)=P_{+}\left(\gamma_{2}, z\right) P_{+}\left(\gamma_{1}, \gamma_{2} z\right) \text {. }
$$

From Theorem 12 in [16, $P_{+}$can be represented in the form

$$
P_{+}= \pm \alpha(z, \lambda)\left(\gamma^{*} \alpha^{-1}(z, \lambda)\right),
$$

where $\alpha: \mathfrak{D} \rightarrow S L(2, \mathbb{C}) / \pm \mathrm{Id}$ is meromorphic. Moreover if $\mathcal{M}$ is non-compact, then $\alpha$ can be chosen holomorphic (see Corollary 4 in [16]). Let $\alpha=\alpha_{+} \alpha_{-}$be the Birkhoff decomposition of $\alpha$. Equation (2.4.5) now implies that $\gamma^{*} \alpha_{-}=\alpha_{-}$and $\gamma^{*} \alpha_{+}=P_{+}^{-1} \alpha_{+}$. Thus $P_{+}= \pm \alpha_{+}\left(\gamma^{*} \alpha_{+}^{-1}\right)$, and we set

$$
C(z, \lambda)=\tilde{C} \cdot \alpha_{+} \text {. }
$$

Then $C$ satisfies $\gamma^{*} C=C$.

Remark 2.10. As pointed out in the proof above, and as known from 9, if $\mathcal{M}$ is a non-compact Riemann surface $\mathcal{M}$, then there exists a holomorphic 1-form, not only a meromorphic 1-form, for every CMC-immersion from $\mathcal{M}$ to $\mathbb{R}^{3}$, i.e., there exists a holomorphic potential on $\mathcal{M}$ for every CMC-immersion from $\mathcal{M}$ to $\mathbb{R}^{3}$.

In general, the monodromy matrix $M_{\gamma}$ in Definition 2.6 is not uniquely determined. We have two possibilities: either the associated family of an immersion $\Psi_{\lambda}$ has umbilic points or the associated family of an immersion $\Psi_{\lambda}$ does not have umbilic points. If $\Psi_{\lambda}$ has umbilic points, then the monodromy matrix $M_{\gamma}$ considered in Definition 2.6 is uniquely determined up to a sign, because the isotropy group of $\Psi_{\lambda}$ consists of $\pm \mathrm{Id}$ only. If $\Psi_{\lambda}$ does not have umbilic points, then the monodromy matrix $M_{\gamma}$ considered in Definition 2.6 is not uniquely determined, because the isotropy group is (in general) not trivial. If we take an element $B_{+}$of the isotropy group, then $M_{\gamma} B_{+}$is also a monodromy matrix in the sense of Definition 2.6.

However, if $\eta$ is an invariant potential on $\mathcal{M}$, meromorphic or holomorphic, then for $\gamma \in \Gamma$ the matrix $M_{\gamma}$ satisfying $\gamma^{*} C=M_{\gamma} C$ is uniquely determined and $\gamma \rightarrow M_{\gamma}$ is a group homomorphism. We are still interested in the more general definition given in Definition [2.6] since in general $M_{\gamma}$ may not be unitary on $S^{1}$, while $M_{\gamma} B_{+}$is unitary on $S^{1}$ for some $B_{+}$in the isotropy group (see e.g. [8]). For more details on this issue we refer to [10].

As pointed out just above, the monodromy matrix $M_{\gamma}$ obtained in Proposition 2.8 is, in general, not unitary on $S^{1}$. However, in order to construct CMCimmersions on $\mathcal{M}=\Gamma \backslash \mathfrak{D}$ we need unitary monodromy (together with the closing conditions). If $M_{\gamma} B_{+}$is unitary, we can continue with the construction of an immersion defined on $\mathcal{M}$. If there is no $B_{+}$in the isotropy group such that $M_{\gamma} B_{+}$ is unitary, then one can try to find at least some dressing transformation, which changes the given monodromy matrix $M_{\gamma} B_{+}$into a unitary monodromy matrix.

Let $0<r<1$ and let $M: A_{r} \rightarrow S L(2, \mathbb{C})$ be holomorphic. Then $M$ is called $s$-unitarizable for $0<r<s<1$ if there exists some $h \in \Lambda_{s} S L(2, \mathbb{C})_{\sigma}$ such that $h M h^{-1} \in \Lambda_{s} S U(2)_{\sigma}$. If $M$ is just defined on the unit circle, then $M$ is called unitarizable if and only if there exists some $h \in \Lambda S L(2, \mathbb{C})_{\sigma}$ such that $h M h^{-1} \in$ $\Lambda S U(2)_{\sigma}$.

Theorem $2.11(9])$. Let $M$ be an element of $\Lambda_{r} S L(2, \mathbb{C})_{\sigma}$. Then $M$ is unitarizable via dressing for some $s \in(r, 1]$ if and only if, for all $\lambda \in S^{1}$,

$$
\operatorname{Tr}(M) \in(-2,2) \text { or } M= \pm \mathrm{Id} \text {. }
$$


As a corollary of the above theorem, for a monodromy matrix of $C$ defined in Proposition 2.8, we have the necessary and sufficient condition for being unitarizable.

Corollary 2.12. We retain the assumptions of Proposition 2.8. Then there exists $h_{+}(\lambda) \in \Lambda_{r}^{+} S L(2, \mathbb{C})_{\sigma}$ such that $\hat{C}=h_{+}(\lambda) C$ is a solution of $d \hat{C}=\hat{C} \eta$, and a monodromy matrix of $\hat{C}$ is $\hat{M}_{\gamma}=h_{+} M_{\gamma} h_{+}^{-1} \in \Lambda_{r} S U(2)_{\sigma}$ if and only if $M_{\gamma}$ satisfies the condition (2.4.6).

Note, if a monodromy matrix for a holomorphic extended framing is unitary, then after Iwasawa splitting, the extended framing admits the same monodromy matrix. By the Sym-Bobenko Formula this yields an immersion $\Psi_{\lambda}$ satisfying $\gamma^{*} \Psi_{\lambda}=R(\lambda) \Psi_{\lambda}(z)$, where $R(\lambda)$ is a rigid motion.

We let $M_{\gamma} \in \Lambda S U(2)_{\sigma}$ be a monodromy matrix of $F$. Then we obtain the following necessary and sufficient condition for the closing of a surface $\Psi_{\lambda=\lambda_{0}}$ with respect to $\gamma$.

Theorem 2.13. We retain the assumptions of Proposition 2.8. Furthermore, if $M_{\gamma} \in \Lambda_{r} S U(2)$, then $M_{\gamma}$ is also a monodromy matrix for $F$ with respect to $\gamma$, where $F$ is the unitary part of the Iwasawa splitting of $C=F W_{+}$. Let $\Psi_{\lambda}$ be defined from $F$ via the Sym-Bobenko Formula (2.2.1). Then $\gamma^{*} \Psi_{\lambda=\lambda_{0}}=\Psi_{\lambda=\lambda_{0}}$ for some $\lambda_{0} \in S^{1}$ holds if and only if

$$
M_{\gamma}\left(\lambda_{0}\right)= \pm \operatorname{Id} \quad \text { and } \quad \partial_{\lambda} M_{\gamma}\left(\lambda_{0}\right)=0
$$

\section{CMC-IMMERSIONS AND TWO COMPLEX VARIABLES}

3.1. Double loop groups and the Iwasawa decomposition. In the context of Wu's Formula [24], it turned out that the extended framings $F(z, \bar{z}, \lambda)$ of some CMC-immersions are restrictions of meromorphic maps $F(z, w, \lambda)$, defined on $\mathfrak{D} \times$ $\overline{\mathfrak{D}}$, where $\mathfrak{D}=$ disk in $\mathbb{C}$ or $\mathbb{C}$ and $\overline{\mathfrak{D}}$ is the complex conjugate domain of $\mathfrak{D}$. Also for the main result of this paper (Theorem 5.8) it will be useful to consider meromorphic extensions to two complex variables.

To explain this in detail one needs to consider double loop groups. Following 14, but interchanging "+" and "-" and " $R$ " and " $r$ ", we set

$$
\mathcal{H}=\Lambda_{r} S L(2, \mathbb{C})_{\sigma} \times \Lambda_{R} S L(2, \mathbb{C})_{\sigma},
$$

where $0<r<R$. Moreover

$$
\begin{aligned}
\mathcal{H}_{+} & =\Lambda_{r}^{+} S L(2, \mathbb{C})_{\sigma} \times \Lambda_{R}^{-} S L(2, \mathbb{C})_{\sigma}, \\
\mathcal{H}_{+, *} & =\Lambda_{r, *}^{+} S L(2, \mathbb{C})_{\sigma} \times \Lambda_{R}^{-} S L(2, \mathbb{C})_{\sigma},
\end{aligned}
$$

$\mathcal{H}_{-}=\left\{\left(g_{1}, g_{2}\right) \in \mathcal{H} ; \quad g_{1}\right.$ and $g_{2}$ extend holomorphically to $A_{r}$ and $\left.\left.g_{1}\right|_{A_{r}}=\left.g_{2}\right|_{A_{r}}\right\}$. We quote Theorem 2.6 in [14].

Theorem 3.1. We have the disjoint union

$$
\mathcal{H}=\bigcup_{n=0}^{\infty} \mathcal{H}_{-} w_{n} \mathcal{H}_{+},
$$

where $w_{n}=\left(\mathrm{Id},\left(\begin{array}{cc}\lambda^{n} & 0 \\ 0 & \lambda^{-n}\end{array}\right)\right)$ if $n=2 k$ and $\left(\mathrm{Id},\left(\begin{array}{cc}0 & \lambda^{n} \\ -\lambda^{-n} & 0\end{array}\right)\right)$ if $n=2 k+1$. The 
loops, for which $n=0$, form an open dense subset of $\mathcal{H}$, and the multiplication map

$$
\mathcal{H}_{-} \times \mathcal{H}_{+, *} \rightarrow \mathcal{H}
$$

is an analytic diffeomorphism onto its image.

We would like to point out that the proof of the theorem above is almost verbatim the proof given in the basic splitting paper [2. Below we show how this implies the well-known $r$-Iwasawa decomposition Theorem 2.2 (see also [12]).

Proof of Theorem 2.2. Let $g \in \Lambda_{r} S L(2, \mathbb{C})_{\sigma}$. Consider the map

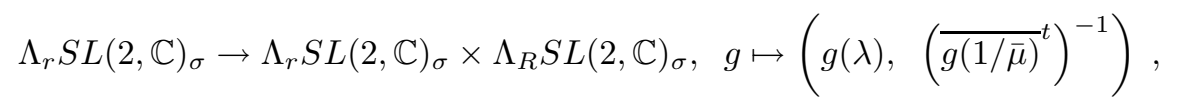

where $R=1 / r,|\lambda|=r$ and $|\mu|=R$. From Theorem 3.1 above we infer that one can write

$$
\left(g(\lambda),\left(\overline{g(1 / \bar{\mu}}^{t}\right)^{-1}\right)=\left(U^{(r)}, U^{(R)}\right)(\mathrm{Id}, \quad W)\left(h_{+}^{(r)}(\lambda), \quad h_{-}^{(R)}(\mu)\right) .
$$

Spelling this out we obtain

$$
\begin{gathered}
g(\lambda)=U^{(r)}(\lambda) h_{+}^{(r)}(\lambda), \\
\left(\overline{g(1 / \bar{\mu}}^{t}\right)^{-1}=U^{(R)}(\mu) W h_{-}^{(R)}(\mu),
\end{gathered}
$$

where $U^{(r)}$ and $U^{(R)}$ denote the boundary values of $U$ on $C_{r}$ and $C_{R}$, respectively. The second equation is equivalent with

$$
g(1 / \bar{\mu})=\left({\overline{U^{(R)}(\mu)}}^{t}\right)^{-1}\left(\bar{W}^{t}\right)^{-1}\left({\overline{h_{-}^{(R)}(\mu)}}^{t}\right)^{-1} .
$$

Replacing $\mu$ by $1 / \bar{\lambda}$ we obtain

$$
g(\lambda)=\left({\overline{U^{(R)}(1 / \bar{\lambda}}}^{t}\right)^{-1}\left(\bar{W}^{t}\right)^{-1}\left({\overline{h_{-}^{(R)}(1 / \bar{\lambda})}}^{t}\right)^{-1} .
$$

A comparison with (3.1.2) shows

$$
\left({\overline{U^{(R)}(1 / \bar{\lambda}}}^{t}\right) U^{(r)}(\lambda)=\left(\bar{W}^{t}\right)^{-1}\left({\overline{h_{-}^{(R)}(1 / \bar{\lambda})}}^{t}\right)^{-1}\left(h_{+}^{(r)}(\lambda)\right)^{-1} .
$$

Equation (3.1.6) is the Birkhoff decomposition of a self-adjoint loop

$$
q(\lambda)=\left({\overline{U^{(R)}(1 / \bar{\lambda}}}^{t}\right) U^{(r)}(\lambda) .
$$

Therefore $W=\mathrm{Id}$, and the right-hand side loop of (3.1.6) is defined on $\mathbb{C} \cup\{\infty\}$. Hence $q(\lambda)$ is constant. Since $q(\lambda)$ is also a positive definite matrix, we obtain $q(\lambda)=k$, where $k$ is a $\lambda$ independent diagonal matrix with entries $k_{0}, k_{0}^{-1}>0$. We set $\tilde{U}=U \tilde{k}$, where $\tilde{k}$ is the $\lambda$ independent diagonal matrix with entries $\sqrt{k_{0}},{\sqrt{k_{0}}}^{-1}$. We have $\left(g(\lambda),\left(\overline{g(1 / \bar{\mu}}^{t}\right)^{-1}\right)=\left(\tilde{U}^{(r)}, \tilde{U}^{(R)}\right)(\mathrm{Id}, \mathrm{Id})\left(\tilde{k}^{-1} h_{+}^{(r)}(\lambda), \tilde{k}^{-1} h_{-}^{(R)}(\mu)\right)$. Moreover, from (3.1.6) we obtain $\tilde{U}^{(r)}(\lambda)=\left({\overline{\tilde{U}^{(R)}(1 / \bar{\lambda})}}^{t}\right)^{-1}$. If $r=1$, the claim follows. Assume now $r<1$. Since $\tilde{U}^{(r)}$ and $\tilde{U}^{(R)}$ are the boundary values of a 
holomorphic function $\tilde{U}$ in $r<|\lambda|<1 / r$, we can restrict the equation above to the unit circle and obtain $\tilde{U}(\lambda)=\left(\overline{\tilde{U}(\lambda)}^{t}\right)^{-1}$, hence $\tilde{U}(\lambda)$ is unitary on $S^{1}$.

3.2. CMC-potentials in the double loop group picture. In this subsection, since the extended framing $F(z, \bar{z}, \lambda)$ of a CMC surface is defined on the unit circle $|\lambda|=1$, we use $r=R=1$. Let $C=C(z, \lambda)=F W_{+}$be a holomorphic extended framing of some CMC-immersion. Using the embedding discussed in the last section we consider

$$
C \rightarrow\left(C,\left(\bar{C}^{t}\right)^{-1}\right)
$$

If $\eta=\eta(z, \lambda)$ denotes the Maurer-Cartan Form of $C, \eta=C^{-1} d C$, then the MaurerCartan Form of the image under the map (3.2.1) is given by

$$
\left(\eta(z, \lambda),-\overline{\eta(z, \lambda)}^{t}\right) \text {. }
$$

We would like to point out that

$$
\left\{\begin{array}{rlr}
\eta(z, \lambda) & =\quad \sum_{k=-1}^{\infty} \eta_{k}(z) \lambda^{k} \\
-\overline{\eta(z, \lambda)}^{t} & =\sum_{k=-1}^{\infty}-\eta_{k}(z) \bar{\lambda}^{k}
\end{array}\right.
$$

3.3. Extended framings in two complex variables. In this subsection, we consider a procedure that is converse to the construction discussed in the previous section. To motivate the approach below we rephrase the second equation of (3.2.3) as

$$
\tau(w, \mu)=-\overline{\eta(\bar{w}, 1 / \bar{\mu})}^{t},
$$

where $w=\bar{z}$ and $\mu=1 / \bar{\lambda}$. Thus

$$
\tau(w, \mu)=\sum_{m=-\infty}^{1} \tau_{m}(w) \mu^{m}
$$

where $\tau_{m}(w)=-{\overline{\eta_{-m}(\bar{w})}}^{t}$. Using the equations above and setting $R=\left(\bar{C}^{t}\right)^{-1}$ we obtain

$$
\left\{\begin{array}{l}
d C=C \eta, \quad C\left(z_{0}, \lambda\right)=\mathrm{Id} \\
d R=R \tau, \quad R\left(\bar{z}_{0}, \lambda\right)=\mathrm{Id}
\end{array}\right.
$$

where and $z_{0}$ and $\bar{z}_{0}$ are the base points chosen in $\mathfrak{D}$ and $\overline{\mathfrak{D}}$, respectively.

We now consider a more general setting, i.e., $w, \mu$ and $\tau(w, \mu)$ are independent of $z, \lambda$ and $\eta(z, \lambda)$, and $z_{0} \in \mathfrak{D}, w_{0} \in \overline{\mathfrak{D}}$ are arbitary, but fixed. Let us start from the potential

$$
\check{\eta}=(\eta(z, \lambda), \quad \tau(w, \mu))=\left(\sum_{k=-1}^{\infty} \eta_{k}(z) \lambda^{k}, \quad \sum_{m=-\infty}^{1} \tau_{m}(w) \mu^{m}\right),
$$

where $z \in \mathfrak{D}, w \in \overline{\mathfrak{D}}, \lambda \in \mathbb{C},|\lambda|=r, \mu \in \mathbb{C}$ and $|\mu|=R$, and $\eta_{k}$ and $\tau_{m}$ are holomorphic differential 1-forms. Let $C$ and $R$ denote the solutions to the differential equations

$$
\left\{\begin{array}{l}
d C=C \eta, \quad C\left(z_{0}, \lambda\right)=\mathrm{Id} \\
d R=R \tau, \quad R\left(w_{0}, \mu\right)=\mathrm{Id}
\end{array}\right.
$$


where $z_{0} \in \mathfrak{D}$ and $w_{0} \in \overline{\mathfrak{D}}$. We consider the generalized Iwasawa decomposition of Theorem 3.1.

$$
(C, R)=(U, U)(\mathrm{Id}, W)\left(V_{+}, V_{-}\right) .
$$

In view of the initial conditions and the fact that the big cell in the double loop group is open, we can assume $W=\mathrm{Id}$, if $(z, w)$ is sufficiently close to $\left(z_{0}, w_{0}\right)$. Thus

$$
C=U^{(r)} V_{+}, \quad R=U^{(R)} V_{-},
$$

and $U=U(z, w, \lambda)$ is meromorphic in two complex variables $z$ and $w$ (see [18]).

3.4. Meromorphic extensions of extended framings. In this section, we show that extended framings have unique meromorphic extensions. This follows essentially from the previous two sections.

Theorem 3.2. Let $F(z, \bar{z}, \lambda), z \in \mathfrak{D}$, be a extended framing of any CMC-immersion. Then there exists a $\lambda$ independent diagonal matrix $l(z, \bar{z}) \in S L(2, \mathbb{C})$, and $F(z, \bar{z}, \lambda) l(z, \bar{z})$ has a meromorphic extension $U(z, w, \lambda)$ to $\mathfrak{D} \times \overline{\mathfrak{D}}$.

Proof. Let $F=F(z, \bar{z}, \lambda)$ be the extended framing of some CMC-immersion. Let $C(z, \lambda)=F(z, \bar{z}, \lambda) W_{+}(z, \bar{z}, \lambda)$ be a holomorphic extended framing. We note that $|\lambda|=1$. Set $R(w, \lambda)=\left(\overline{C(\bar{w}, \lambda)}^{t}\right)^{-1}$, where $w \in \overline{\mathfrak{D}}$ is independent of $z$. Then the pair $(C(z, \lambda), R(w, \lambda))$ corresponds to a potential $(\eta, \tau)$ as considered in the previous section:

$$
\eta(z, \lambda)=C^{-1} d C, \quad \tau(w, \lambda)=R^{-1} d R .
$$

We thus obtain $C(z, \lambda)$ and $R(w, \lambda)$ as the solutions to $d C=C \eta, z \in \mathfrak{D}$, and $d R=R \tau, w \in \overline{\mathfrak{D}}$, where we also use $C\left(z_{0}, \lambda\right)=\mathrm{Id}$ and $R\left(w_{0}=\bar{z}_{0}, \lambda\right)=\mathrm{Id}$. As in the previous section we obtain (using the unique generalized Iwasawa decomposition of Theorem 3.1, i.e., $\left.\mathcal{H}_{-} \times \mathcal{H}_{+, *} \rightarrow \mathcal{H}\right)$

$$
\left\{\begin{array}{l}
C(z, \lambda)=U(z, w, \lambda) V_{+}(z, w, \lambda) \\
R(w, \lambda)=U(z, w, \lambda) V_{-}(z, w, \lambda)
\end{array}\right.
$$

Therefore

$$
\begin{aligned}
U(z, w, \lambda) & =C(z, \lambda) V_{+}(z, w, \lambda)^{-1} \\
& =\quad R(w, \lambda) V_{-}(z, w, \lambda)^{-1} .
\end{aligned}
$$

Substituting $C(z, \lambda)=F(z, \bar{z}, \lambda) W_{+}(z, \bar{z}, \lambda)$ and

$$
R(w, \lambda)=\left(\overline{C(\bar{w}, \lambda)}^{t}\right)^{-1}=F(\bar{w}, w, \lambda)\left({\overline{W_{+}(\bar{w}, w, \lambda)}}^{t}\right)^{-1},
$$

we obtain

$$
\begin{aligned}
U(z, w, \lambda) & =F(z, \bar{z}, \lambda) W_{+}(z, \bar{z}, \lambda) V_{+}(z, w, \lambda)^{-1} \\
& =F(\bar{w}, w, \lambda)\left({\overline{W_{+}(\bar{w}, w, \lambda}}^{t}\right)^{-1} V_{-}(z, w, \lambda)^{-1} .
\end{aligned}
$$

For $w=\bar{z}$, 3.4.3) implies that

$$
W_{+}(z, \bar{z}, \lambda) V_{+}(z, \bar{z}, \lambda)^{-1}=\left({\overline{W_{+}(z, \bar{z}, \lambda}}^{t}\right)^{-1} V_{-}(z, \bar{z}, \lambda)^{-1} .
$$

The left-hand side is in $\Lambda^{+} S L(2, \mathbb{C})_{\sigma}$, and the right-hand side is in $\Lambda^{-} S L(2, \mathbb{C})_{\sigma}$, thus $W_{+} V_{+}^{-1}=\left({\overline{W_{+}}}^{t}\right)^{-1} V_{-}^{-1}=l(z, \bar{z})$, where $l$ is a $\lambda$ independent diagonal matrix with entries $l_{0}, l_{0}^{-1}>0$. We note that $l(z, \bar{z})^{2}=V_{-, 0}^{-1}(z, \bar{z})$ by (3.4.4), where $V_{-, 0}$ is 
the first coefficient matrix of the expansion of $V_{-}(z, \bar{z}, \lambda)$ with respect to $\lambda$. From (3.4.3), we have

$$
U(z, \bar{z}, \lambda)=F(z, \bar{z}, \lambda) l(z, \bar{z}) .
$$

Therefore $F(z, \bar{z}, \lambda) l(z, \bar{z})$ has a meromorphic extension $U(z, w, \lambda)$ to $\mathfrak{D} \times \overline{\mathfrak{D}}$.

Remark 3.3. In general, there will be many meromorphic extensions of $F \cdot l$ to $\mathfrak{D} \times \overline{\mathfrak{D}}$. However, if $U$ and $T$ are both such meromorphic extensions to $\mathfrak{D} \times \overline{\mathfrak{D}}$ and also satisfy (3.4.1), then $U=T$, since the generalized Iwasawa decomposition of Theorem 3.1 is unique.

The meromorphic extension $U(z, w, \lambda)$ constructed in the proof will be called "the unique meromorphic extension" of $F \cdot l$.

Remark 3.4. In view of the Sym-Bobenko Formula for CMC-immersions it is tempting to consider the 1-parameter family of complex surfaces (with singularities)

$$
\Psi_{\lambda}=\left.\left(\frac{\mathrm{d}}{\mathrm{dt}} U(z, w, \lambda) \cdot U(z, w, \lambda)^{-1}+U(z, w, \lambda) \cdot \frac{i}{2} \sigma_{3} U(z, w, \lambda)^{-1}\right)\right|_{\lambda=e^{i t}}
$$

where $U(z, w, \lambda)$ is the meromorphic extension of the extended framing $F(z, \bar{z}, \lambda) l(z, \bar{z})$. Even for arbitary potentials $(\eta(z) d z, \tau(w) d w)$, we expect these immersions to be of great interest to the theory of integrable surface equations. We plan to pursue this topic in a separate publication [11.

\section{Natural potentials For CMC-IMmersions}

4.1. Existence of skew-hermitian potentials. In this section we consider a new type of potentials, so-called "skew-hermitian potentials", derived from a CMCimmersion from $\mathcal{M}=\Gamma \backslash \mathfrak{D}$ to $\mathbb{R}^{3}$. These potentials are meromorphic 1-forms on D. From (3.4.1), we obtain the equation

$$
C(z, \lambda)=U(z, w, \lambda) \cdot V_{+}(z, w, \lambda)
$$

where $(z, w) \in \mathfrak{D} \times \overline{\mathfrak{D}}$ are independent variables and $U(z, w, \lambda)$ is the unique meromorphic extension of the extended framing $F(z, \bar{z}, \lambda) l(z, \bar{z})$. We note that in (4.1.1) we can replace $w$ by any function of $z$ and $\bar{z}$. First we set $w=z$, then the entries of $U(z, z, \lambda)=C(z, \lambda) V_{+}(z, z, \lambda)^{-1}$ are meromorphic functions with respect to $z$. Thus, $U(z, z, \lambda)$ can be considered as a meromorphic extended framing, which is obtained from $C$ by gauging with some $V_{+}{ }^{-1}$. Moreover, for $z \in \mathbb{R} \cap \mathfrak{D}$, we have $F(z, \bar{z}, \lambda) l(z, \bar{z})=U(z, z, \lambda)$. Therefore

$$
\begin{aligned}
\alpha=F^{-1} d F & =l U^{-1} d U l^{-1}-(d l) \cdot l^{-1} \\
& =\left(\begin{array}{cc}
u_{11} & l_{0}^{2} u_{12} \\
l_{0}^{-2} u_{21} & u_{22}
\end{array}\right)-\left(\begin{array}{cc}
l_{0}^{\prime} / l_{0} & 0 \\
0 & -l_{0}^{\prime} / l_{0}
\end{array}\right), \text { for } z \in \mathbb{R} \cap \mathfrak{D},
\end{aligned}
$$

where $u_{i j},\{i, j\}=\{1,2\}$, are the entries of $U^{-1} d U$ and $l=\operatorname{diag}\left(l_{0}, l_{0}^{-1}\right)$. We note that $l_{0}^{\prime} / l_{0}$ is half of the logarithmic derivative of $l_{0}^{2}$, and $l_{0}^{2}$ can be extended to all $(z, w) \in \mathfrak{D} \times \overline{\mathfrak{D}}$ (see the proof of Theorem 3.2). Therefore the right-hand side of (4.1.2) can be extended to all $z \in \mathfrak{D}$. Clearly $U(z, z, \lambda) l(z, \bar{z})^{-1}$ is a unitary matrix for $z \in \mathbb{R} \cap \mathfrak{D}$. We denote by $\zeta$ the unique extension of $\alpha$, which is obtained by choosing the natural meromorphic extension of (4.1.2) via the unique meromorphic extension of $U l^{-1}$. Therefore we have proved the following. 
Theorem 4.1 (Existence of skew-hermitian potentials). Let $\mathcal{M}=\Gamma \backslash \mathfrak{D}$ be $a$ connected Riemann surface with universal cover $\mathfrak{D}$ and Fuchsian group $\Gamma$. Assume that $\Psi: \mathcal{M} \rightarrow \mathbb{R}^{3}$ is an immersion of constant mean curvature $H=1 / 2$ with associated family $\Psi_{\lambda}: \mathfrak{D} \rightarrow \mathbb{R}^{3}$. Then $\Psi_{\lambda}$ can be derived from a meromorphic 1 -form $\zeta$ on $\mathfrak{D}$ such that

$$
\zeta(z, \lambda)=\lambda^{-1} \zeta_{-1}(z)+\zeta_{0}(z)+\lambda \zeta_{1}(z)
$$

where each $\zeta_{j}, j=-1,0,1$, is a meromorphic 1 -form on $\mathfrak{D}$ and $\zeta$ is skew-hermitian for $z \in \mathbb{R} \cap \mathfrak{D}$.

Potentials of the form just stated will be called skew-hermitian potentials.

Remark 4.2. We note that Theorem 4.1 implies that the Maurer-Cartan form $\alpha=$ $F^{-1} d F$ of some extended framing $F$ has a unique meromorphic extension, while the extended framing $F$ may not have a unique meromorphic extension.

4.2. Periods of skew-hermitian potentials. We have shown in Theorem 3.2 that $F(z, \bar{z}, \lambda)$ has, up to a $\lambda$-independent diagonal factor, a meromorphic extension $U(z, w, \lambda)$ to $\mathfrak{D} \times \overline{\mathfrak{D}}$. Moreover, the square of this diagonal factor has a meromorphic extension to $\mathfrak{D} \times \overline{\mathfrak{D}}$ as well. Hence if $\alpha$ is the Maurer-Cartan form of the extended coordinate framing of some CMC-immersion, then $\alpha(z, \bar{z}, \lambda)$ has a meromorphic extension $\hat{\zeta}(z, w, \lambda)$. We set $w=z$; then the corresponding skew-hermitian potential $\zeta=\hat{\zeta}(z, w=z, \lambda)$ is

$$
\zeta(z, z, \lambda)=\left\{\lambda^{-1}\left(\begin{array}{cc}
0 & h(z) \\
\frac{Q(z)}{h(z)} & 0
\end{array}\right)+\zeta_{0}+\lambda\left(\begin{array}{cc}
0 & -\frac{\overline{Q(\bar{z})}}{h(z)} \\
-h(z) & 0
\end{array}\right)\right\} d z,
$$

where $h(z)=\left.\exp (1 / 2 u(z, w))\right|_{w=z}=l_{0}^{2}(z, z)$ with $l_{0}$ defined in the proof of Theorem 3.2. which is the unique extension of the square root of the conformal factor $e^{u(z, \bar{z})}$ of a CMC-immersion. We note that $h(z)$ is real for $z \in \mathfrak{D} \cap \mathbb{R}$. Also, $Q$ is the coefficient of the Hopf differential of the CMC immersion and

$$
\zeta_{0}=\left(\begin{array}{cc}
\left.\frac{1}{4}\left(u_{z}(z, w)-u_{w}(z, w)\right)\right|_{w=z} & 0 \\
0 & -\left.\frac{1}{4}\left(u_{z}(z, w)-u_{w}(z, w)\right)\right|_{w=z}
\end{array}\right) .
$$

Corollary 4.3. If $u$ has real coefficients, i.e., if $u(z, \bar{z})=u(\bar{z}, z)$, then $\zeta_{0}(z, z)=0$.

Clearly if the fundamental group $\Gamma=\pi_{1}(\mathcal{M})$ contains the real translation $z \rightarrow$ $z+p$, then the conformal factor $e^{u(z, \bar{z})}$ and the coefficient $Q$ of the Hopf differential of the CMC-immersion are periodic with period $p$.

Corollary 4.4. If the fundamental group $\Gamma=\pi_{1}(\mathcal{M})$ contains the real translation $z \rightarrow z+p, p \in \mathbb{R}$, then the corresponding skew-hermitian potential $\zeta$ defined in (4.2.1) is periodic with period $p$.

4.3. Uniqueness of skew-hermitian potentials. We know that normalized potentials are uniquely determined while holomorphic potentials are not (see the Introduction and [13]). It is thus natural to ask whether the new type of potential defined above is uniquely determined by a given CMC-immersion. From Section 4.2. we will consider the skew-hermitian potentials defined in (4.2.1). The following theorem implies that the skew-hermitian potential is almost uniquely determined by a CMC-immersion. 
Theorem 4.5. Let $\zeta=\lambda^{-1} \zeta_{-1}+\zeta_{0}+\lambda \zeta_{1}, \lambda \in S^{1}$, be a skew-hermitian potential defined in (4.2.1). Assume that the same assumptions hold for $\hat{\zeta}$. Then $\zeta$ and $\hat{\zeta}$ induce the same CMC-immersion if and only if $\hat{\zeta}=L_{0}^{-1} \zeta L_{0}+L_{0}^{-1} d L_{0}$, where $L_{0}(z)= \pm \operatorname{Id}$ or $L_{0}(z)=\operatorname{diag}( \pm i, \mp i)$.

Proof. " $\Rightarrow$ " Since $\zeta$ and $\hat{\zeta}$ are meromorphic potentials for the same immersion, we know that the solutions to $d C=C \zeta$ and $d \hat{C}=\hat{C} \hat{\zeta}$ satisfy the relation

$$
\hat{C}=C L_{+},
$$

where $L_{+} \in \Lambda^{+} S L(2, \mathbb{C})_{\sigma}$ is meromorphic in $z \in \mathfrak{D}$ and holomorphic for $\lambda \in \mathbb{C}$.

For $z \in \mathbb{R} \cap \mathfrak{D}$ we know that $L_{+}(z, \lambda)$ is unitary. Hence $\left({\overline{L_{+}(z, \lambda)}}^{t}\right)^{-1}=L_{+}(z, \lambda)$ for $z \in \mathbb{R} \cap \mathfrak{D}$. Therefore, $L_{+}(z, \lambda)=L_{0}(z)$ is independent of $\lambda$ for $z \in \mathbb{R} \cap \mathfrak{D}$, and $\hat{C}=C L_{0}$ implies $\hat{\zeta}=L_{0}^{-1} \zeta L_{0}+L_{0}^{-1} d L_{0}$ for $z \in \mathbb{R} \cap \mathfrak{D}$, where $L_{0}=\operatorname{diag}\left(l_{0}, l_{0}^{-1}\right)$ and $\left|l_{0}\right|=1$. Moreover $\hat{h}(z)$, which is an entry of $\hat{\zeta}$ defined by (4.2.1), is real for $z \in \mathfrak{D} \cap \mathbb{R}$, and thus $L_{+}= \pm \operatorname{Id}$ or $L_{+}=\operatorname{diag}( \pm i, \mp i)$, and by analytic continuation, $L_{+}= \pm \mathrm{Id}$ or $\operatorname{diag}( \pm i, \mp i)$ for all $z \in \mathfrak{D}$.

" $\Leftarrow$ " Let $L_{0}= \pm \operatorname{Id}$ or $\operatorname{diag}( \pm i, \mp i)$. We set $\hat{C}=C L_{0}, \hat{\zeta}=\hat{C}^{-1} d \hat{C}$ and $\zeta=$ $C^{-1} d C$. We consider the Iwasawa decomposition of $\hat{C}=(F \tilde{U})\left(\tilde{U}^{-1} W_{+} L_{0}\right)$, where $C=F W_{+}$is the Iwasawa decomposition of $C$ and $\tilde{U} \in U(1) . \tilde{U}$ goes away in the Sym-Bobenko Formula, thus $F \tilde{U}$ and $F$ define the same CMC surface.

\section{CMC-CYLINDERS}

5.1. Necessary and sufficient condition for CMC surfaces with a period. In this section, we consider CMC-cylinders. These are homeomorphic to $\mathbb{S}^{2} \backslash$ $\left\{p_{1}, p_{2}\right\}$, where $p_{1}$ and $p_{2}$ are different, but otherwise arbitary, points in $\mathbb{S}^{2}$. Using a Möbius transformation, we can move these two points to the north pole and the south pole of $\mathbb{S}^{2}$, and using stereographic projection from the north pole we can assume that every CMC-cylinder is an immersion from $\mathcal{M}=\mathbb{C} \backslash\{0\}$ to $\mathbb{R}^{3}$. Then using a change of coordinates we can assume that every CMC-cylinder is an immersion from $\mathcal{M}=\mathbb{C} / p \mathbb{Z}$, where $p$ is a real number. Since the fundamental group of a CMC-cylinder is generated by a single period, $p \in \mathbb{R}$, we can, by Corollary 4.4. assume that the corresponding skew-hermitian potential is well defined on $\mathcal{M}=C / p \mathbb{Z}$.

Proposition 5.1. Let $\zeta=\lambda^{-1} \zeta_{-1}+\zeta_{0}+\lambda \zeta_{1}, \lambda \in S^{1}$, be a meromorphic potential on $\mathfrak{D}=\mathbb{C}$ such that $\zeta$ is skew-hermitian for $z \in \mathbb{R}$. Let $C(z, \lambda)$ be the unique solution to $d C=C \zeta, C(0, \lambda)=\mathrm{Id}$. Assume $\zeta$ is periodic with period $p \in \mathbb{R}$. Then the $\mathrm{CMC}$ surface associated with $\zeta$ for $\lambda=1$ has the translation by $p \in \mathbb{R}$ in its fundamental group if and only if the unitary monodromy matrix $C(p, \lambda)$ satisfies the closing conditions (2.4.7) for $\lambda=1$.

Proof. Let $C$ be the solution to $d C=C \zeta, C(0, \lambda)=\mathrm{Id}$, where $0 \in \mathfrak{D}=\mathbb{C}$. Since $\zeta$ is skew-hermitian for $z \in \mathbb{R}$ we obtain that $C(z, \lambda) \in \Lambda_{r} S U(2)_{\sigma}$ for $z \in \mathbb{R}$. Since $\zeta$ is periodic with period $p$ we have $C(p+z, \lambda)=\chi(\lambda) C(z, \lambda)$ by Proposition 2.8. The initial condition $C(0, \lambda)=\operatorname{Id}$ implies $\chi(\lambda)=C(p, \lambda)$. Since $p$ is a real number, $\chi(\lambda)$ is a unitary matrix, and thus $\chi(\lambda)=C(p, \lambda)$ is also a monodromy matrix for $F(z, \bar{z}, \lambda)$, which is the unitary part of the Iwasawa decomposition of $C(z, \lambda)=F(z, \bar{z}, \lambda) W_{+}(z, \bar{z}, \lambda)$. The claim now follows from Theorem 2.13. 
Corollary 5.2. We retain the assumptions of Proposition [5.1. Then, for a cylinder, $C(z, 1)$ and $F(z, \bar{z}, 1)$ are periodic with period $p$.

Now we will give the definition of a frame periodic surface.

Definition 5.3. We call a surface frame periodic for $\lambda=\lambda_{0} \in S^{1}$ if the frame $F\left(z, \bar{z}, \lambda=\lambda_{0}\right) \in S U(2)$ of a CMC-immersion $\Psi_{\lambda=\lambda_{0}}$ is periodic with period $p$.

Corollary 5.4. We retain the assumptions of Proposition 5.1. Then a surface is frame periodic at $\lambda=\lambda_{0} \in S^{1}$ if and only if $C\left(z, \lambda_{0}\right)$ is periodic.

5.2. Frame periodic CMC-immersions. To illustrate the discussion above, we consider the construction of a frame periodic surface from a skew-hermitian periodic potential. It turns out that it is easy to modify the "generalized Weierstrass representation" (13]) so that the first closing condition for a monodromy matrix is trivially satisfied, i.e., $\left.M\right|_{\lambda=1}= \pm \mathrm{Id}$. To motivate our approach, assume first that $\zeta$ is the skew-hermitian potential as in (4.2.1) associated with a frame periodic surface. Let $C$ be the solution to $d C=C \zeta, C(0, \lambda)=\operatorname{Id}$. Then $C(p, \lambda)$ is a unitary monodromy matrix and $C_{0}(z)=C(z, \lambda=1)$ is periodic.

We use this last fact as a starting point for a "sufficiently nice potential". By the remark just made, every frame periodic CMC surface can be obtained in this way. Let us start, conversely, from a meromorphic and periodic matrix $C_{0}(z)$ of the form

$$
C_{0}(z)=\left(\begin{array}{cc}
\frac{a_{0}(z)}{-\overline{b_{0}(\bar{z})}} & \frac{b_{0}(z)}{a_{0}(\bar{z})}
\end{array}\right)
$$

where $a_{0}(z), b_{0}(z)$ are periodic functions of period $p \in \mathbb{R}$ satisfying $\operatorname{det} C_{0}(z)=$ $a_{0}(z) \overline{a_{0}(\bar{z})}+b_{0}(z) \overline{b_{0}(\bar{z})}=1$. In particular $C_{0}$ is unitary for $z \in \mathbb{R}$. Set

$$
\begin{aligned}
\zeta_{0}(z) & =c \\
& =\left(\begin{array}{cc}
\nu(z) & C_{0}^{-1} C_{0}^{\prime} \\
-\overline{\kappa(\bar{z})} & -\nu(z)
\end{array}\right),
\end{aligned}
$$

where $\nu$ and $\kappa$ are periodic 1 -forms of period $p \in \mathbb{R}$ and $\overline{\nu(\bar{z})}=-\nu(z)$. We take meromorphic 1-forms $h(z)$ and $\overline{g(\bar{z})}$ on $\mathbb{C}$, which are periodic of period $p$, and satisfy $\kappa(z)=h(z)-\overline{g(\bar{z})}$ for $z \in \mathbb{C}$. Set

$$
\zeta(z, \lambda)=\left\{\lambda^{-1}\left(\begin{array}{cc}
0 & h(z) \\
g(z) & 0
\end{array}\right)+\left(\begin{array}{cc}
\nu(z) & 0 \\
0 & -\nu(z)
\end{array}\right)+\lambda\left(\begin{array}{cc}
0 & -\overline{g(\bar{z})} \\
-\overline{h(\bar{z})} & 0
\end{array}\right)\right\} d z .
$$

Then $\zeta(z, \lambda=1)=\zeta_{0}(z)$ and $\zeta$ is meromorphic for $z \in \mathfrak{D}$ and skew-hermitian for $z \in \mathbb{R}$. Therefore $\zeta$ satisfies the assumptions of Proposition 5.1. Let $C=C(z, \lambda)$ denote the solution to $d C=C \zeta, C(0, \lambda)=\mathrm{Id}$. Our construction implies $C(z, 1)=$ $C_{0}(z)$, whence the first closing condition is satisfied, i.e., $\chi(1)= \pm$ Id. Therefore we have shown

Theorem 5.5. Let $C_{0}(z)$ be given by (5.2.1) and assume $C_{0}(z)$ is periodic with period p. Set $\zeta_{0}=C_{0}^{-1} d C_{0}$ and define $\zeta$ by (5.2.3). Then $\zeta$ satisfies the assumptions of Proposition 5.1 and defines a frame periodic CMC-immersion. Moreover, every frame periodic CMC-immersion can be obtained this way.

Remark 5.6. The construction of $\zeta$ from $\zeta_{0}$ carried out above shows that there is a lot of freedom. Actually, different choices of $h$ and $g$ yield by and large different surfaces. 
5.3. Second closing conditions. Finally, we consider CMC-cylinders. By the discussion of the last section it only remains to consider the second closing condition, i.e., $\left.\partial_{\lambda} M\right|_{\lambda=1}=0$, where $M$ is the monodromy matrix defined in the sense of Definition 2.6. There is a nice trick reducing the second closing condition to the vanishing of some integral [19, 20].

Proposition 5.7. If $C_{0}(z)$ is as in (5.2.1) and if $\zeta$ is defined as in (5.2.3), then $\zeta$ satisfies the assumptions of Proposition 5.1 and the first closing condition, i.e., $\left.M\right|_{\lambda=1}= \pm \mathrm{Id}$, is satisfied. The second closing condition, i.e., $\left.\partial_{\lambda} M\right|_{\lambda=1}=0$, is satisfied if and only if

$$
\int_{0}^{p}\left\{\left.C_{0} \partial_{\lambda} \zeta\right|_{\lambda=1} C_{0}^{-1}\right\} d w=0
$$

We summarize the discussion of the last few sections.

Theorem 5.8. The construction outlined in Sections 5.2 and 5.3 produces all frame periodic $\mathrm{CMC}$-immersions. Moreover, the construction above also produces all CMC-cylinders.

5.4. New examples of CMC-cylinders. Below we illustrate how the theory presented above can be applied to concrete constructions of CMC-cylinders. Let $C_{0}$ be the following matrix:

$$
C_{0}=\left(\begin{array}{cc}
\cos (z) & \sin (z) \\
-\sin (z) & \cos (z)
\end{array}\right) .
$$

Clearly $C_{0}$ is holomorphic in $\mathbb{C}$, periodic with period $2 \pi$ and unitary for $z \in \mathbb{R}$. Then for the Maurer-Cartan form $\zeta_{0}, \zeta_{0}=C_{0}^{-1} d C_{0}$ of $C_{0}$, we obtain

$$
\zeta_{0}=\left(\begin{array}{cc}
0 & 1 \\
-1 & 0
\end{array}\right) \text {. }
$$

We decompose $1=h(z)-\overline{g(\bar{z})}=1 / 2 e^{i k \cos (z)}+1 / 2\left(2-e^{i k \cos (z)}\right)$ as in Section 5.2. and we introduce $\lambda$ according to (5.2.3). Then we obtain the periodic skewhermitian potential

$$
\zeta(z, \lambda)=\left(\begin{array}{cc}
0 & h(z) \lambda^{-1}-\overline{g(\bar{z})} \lambda \\
g(z) \lambda^{-1}-\overline{h(\bar{z})} \lambda & 0
\end{array}\right),
$$

where $h(z)=1 / 2 e^{i k \cos (z)}, g(z)=-1 / 2\left(2-e^{-i k \cos (z)}\right)$ and $k$ is some real number. To determine $k$, we evaluate (5.3.1):

$$
\int_{0}^{2 \pi}\left\{\left.C_{0} \partial_{\lambda} \zeta\right|_{\lambda=1} C_{0}^{-1}\right\} d w=\left(\begin{array}{cc}
0 & 2 \pi J_{2}(k) \\
2 \pi J_{2}(k) & 0
\end{array}\right),
$$

where $J_{2}(z)$ is the Bessel function of the first kind (see [3]). If we choose $k$ such that $J_{2}(k)=0$, then we obtain an example of a CMC-cylinder. In Figure 1 we have the CMC-cylinder corresponding to two different values of $k$. In the left picture in Figure 1, we use $k=5.13$, the square domain $0<x<2 \pi$ and $-0.4<y<0.4$. In the right picture in Figure 1. we use $k=8.41$, the square domain $0<x<2 \pi$ and $-0.1<y<0.1$, where $z=x+i y$.

Remark 5.9. (1) In [19], 20], many examples are given starting from skew-hermitian potentials. However a comparsion of the Hopf differentials of these surfaces with the example above shows that the surface given above is different from the examples given in [19] and [20] and therefore yields a new example of a CMC-cylinder. 

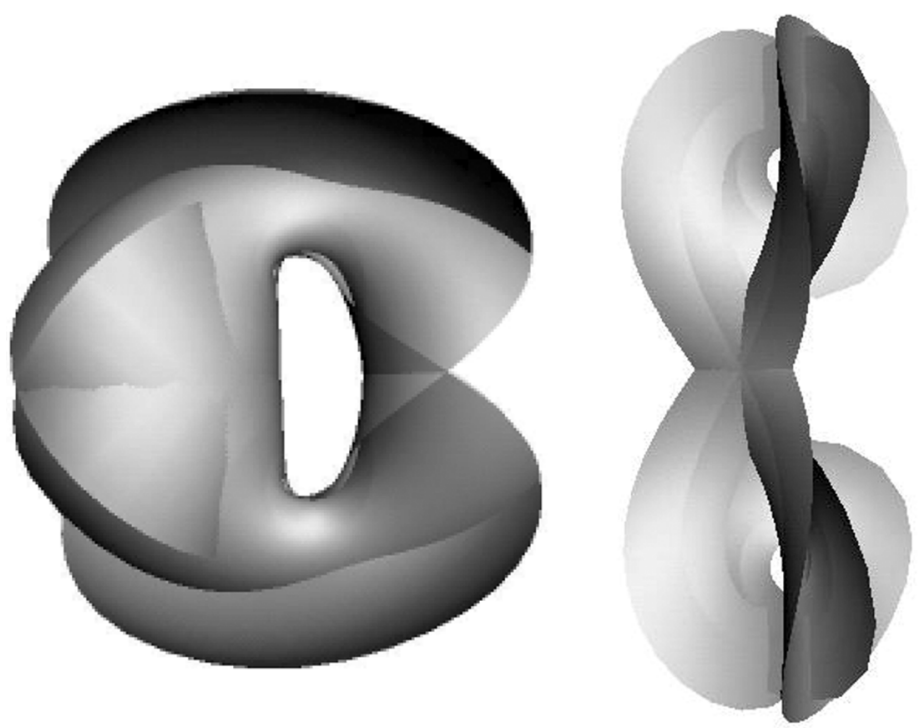

Figure 1. New example of a CMC-cylinder. Figures are constructed using 23 .

(2) The main result of this paper shows how one can construct CMC-cylinders from skew-hermitian matrices, and even better, from matrices of type $C_{0}$. Theorem 4.5 shows that different potentials yield (by and large) different immersions. A fine classification of CMC-cylinders would be highly desirable.

\section{ACKNOWLEDGEMENTS}

We would like to thank Ivan Sterling for making us aware of the fact that our argument of Section 5.1 actually does apply to all (conformal) CMC cylinders.

\section{REFERENCES}

[1] A. I. Bobenko, Constant mean curvature surfaces and integrable equations, Russian Math. Surveys 46 (1991), no. 4, 1-45. MR1138951(93b:53009)

[2] M. Bergvelt, M. Guest, Actions of loop groups on harmonic maps, Trans. Amer. Math. Soc. 326 (1991), 861-886. MR 1062870 (91k:58022)

[3] F. Bowman, Introduction to Bessel Functions, Dover, New York, 1958. MR0097539 (20:4007)

[4] E. A. Coddington, N. Levinson, Theory of ordinary differential equations, McGraw-Hill, New York, 1955. MR0069338 (16:1022b)

[5] J. Dorfmeister, Generalized Weierstrass representations of surfaces, Surveys on Geometry and Integrable Systems, Advanced Studies in Pure Mathematics, to appear.

[6] J. Dorfmeister, G. Haak, Meromorphic potentials and smooth surfaces of constant mean curvature, Math. Z. 224 (1997), 603-640. MR1452051 (98c:53009)

[7] J. Dorfmeister, G. Haak, On symmetries of constant mean curvature surfaces, PartII: Symmetries in a Weierstrass-type representation, Int. J. Math., Game Theory Algebra 10 (2000), 121-146. MR1784970 (2001k:53011)

[8] J. Dorfmeister, G. Haak, On constant mean curvature surfaces with periodic metric, Pacific J. Math. 182 (1998), 229-287. MR1609603 (99f:53007)

[9] J. Dorfmeister, G. Haak, Construction of non-simply connected CMC surfaces via dressing, J. Math. Soc. Japan 55 (2003), no. 2, 335-364. MR1961290 (2004d:53010)

[10] J. Dorfmeister, M. Kilian, Dressing preserving the fundamental group, preprint. 
[11] J. Dorfmeister, S.-P. Kobayashi, F. Pedit, Complex surfaces of constant mean curvature fibered by minimal surfaces, in preparation.

[12] J. Dorfmeister, I. Mcintosh, F. Pedit, H. Wu, On the meromorphic potential for a harmonic surface in a k-symmetric space, Manuscripta Math. 92 (1997), no. 2, 143-152. MR 1428645 (98h:58051)

[13] J. Dorfmeister, F. Pedit and H. Wu, Weierstrass type representation of harmonic maps into symmetric spaces, Comm. Anal. Geom. 6 (1998), no. 4, 633-668. MR1664887(2000d:53099)

[14] J. Dorfmeister, H. Wu, Constant mean curvature surfaces and loop groups, J. Reine Angew. Math. 440 (1993), 43-76. MR 1225957 (94j:53005)

[15] M. A. Guest, Harmonic maps, loop groups, and integrable systems, London Mathematical Society Student Texts 38, Cambridge University Press, 1997. MR.1630443 (99g:58036)

[16] R. C. Gunning, Lectures on Riemann surfaces, Princeton Mathematical Notes, Princeton University Press, 1966. MR0207977 (34:7789)

[17] J.-P. Kahane, Series de Fourier absolument convergentes, Springer-Verlag, 1970. MR0275043 $(43: 801)$

[18] L. Kaup, B. Kaup, Holomorphic functions of several variables, de Gruyter Studies in Mathematics 3, Walter de Gruyter \& Co. 1983. MR0716497 (85k:32001)

[19] M. Kilian, Constant mean curvature cylinders, Doctoral Thesis, Univ. of Massachusetts (Amherst), U.S.A., September 2000.

[20] M. Kilian, I. Mcintosh, N. Schmitt, New constant mean curvature surfaces, Experiment. Math. 9 (2000), no. 4, 595-611. MR1806295 (2002e:53008)

[21] A. Pressley and G. Segal, Loop Groups, Oxford Mathematical Monographs, Oxford University Press, 1986. MR0900587 (88i:22049)

[22] N. Schmitt, Constant mean curvature trinoids, preprint.

[23] N. Schmitt, CMCLab, http://www.gang.umass.edu/software.

[24] H. Wu, A simple way for determining the normalized potentials for harmonic maps, Ann. Global Anal. Geom. 17 (1999), 189-199. MR1675409 (2000g:58022)

Zentrum Mathematik, Technische Universität München Boltzmannstr. 3, D-85747, Garching, Germany

E-mail address: dorfm@ma.tum.de

School of Information Environment, Tokyo Denki University Muzai Gakuendai 21200 InZai, Chiba 270-1382, Japan

E-mail address: shimpei@sie.dendai.ac.jp 